\title{
Evolution of care pathways for babies with hypoplastic left heart syndrome: integrating mechanistic and clinical process investigation, standardization, and collaborative study
}

\author{
Tara Karamlou, Hani K. Najm \\ Division of Pediatric Cardiac Surgery, Cleveland Clinic Heart Vascular Institute, Cleveland, OH, USA \\ Contributions: (I) Conception and design: All authors; (II) Administrative support: HK Najm; (III) Provision of study materials or patients: None; \\ (IV) Collection and assembly of data: T Karamlou; (V) Data analysis and interpretation: All authors; (VI) Manuscript writing: All authors; (VII) Final \\ approval of manuscript: All authors. \\ Correspondence to: Tara Karamlou, MD, MSc. Associate Professor of Surgery, Division of Pediatric Cardiac Surgery, Director of Research and \\ Education, Cleveland Clinic, Heart Vascular Institute, M41-022A, 9500 Euclid Avenue, Cleveland, OH, USA. Email: karamlt@ccf.org.
}

\begin{abstract}
Since initial descriptions of staged palliation for hypoplastic left heart syndrome (HLHS) in the 1980's, much has been learned about the pathophysiology of the single ventricle circulation. New therapies that leverage systems biology and clinical derivatives have been developed. While in-hospital mortality and morbidity for babies with HLHS have continued to improve, there remains a long (and daresay winding) road ahead to achieve ideal outcomes. Important variation in even these abbreviated in-hospital metrics persists among institutions and currently utilized prediction models explain only a small amount of this variation. Moreover, long-term survival and neurodevelopmental health for patients with HLHS are infrequently reported and remain suboptimal despite improved in-hospital outcomes. This focused review will describe the evolution of national outcomes for HLHS over time and the potential factors motivating improved time-related mortality. Emerging modifiable risk-factors that hold promise in terms of moving the needle for long-term success, including social determinants of health and the delineation of genetic profiles, will be discussed. Specifically, this review will integrate contemporary data based on the first murine HLHS models that suggest a genetically elicited modular phenotype with environmental factors known to impact the initial durability of surgical therapies. A comprehensive approach to the management of HLHS, which leverages both proactive transplantation and hybrid palliation, in addition to traditional Norwood palliation, will be emphasized to extend and match management to the complete spectrum of patient riskprofiles. Finally, we will explore the critical role that national collaboratives and quality reporting initiatives have played in improving outcomes and shifting the focus to more meaningful long-term survival and neurodevelopment.
\end{abstract}

Keywords: Congenital heart disease (CHD); single ventricle; outcomes; hypoplastic left heart

Submitted Sep 18, 2019. Accepted for publication Oct 12, 2019.

doi: $10.21037 /$ jtd.2019.10.75

View this article at: http://dx.doi.org/10.21037/jtd.2019.10.75

\section{Introduction}

The first 'long-term' survivor of the Norwood procedure, a $3.7 \mathrm{~kg}$ newborn with aortic atresia and mitral atresia and the absence of a left ventricular cavity, was chronicled by Norwood and colleagues in 1983 (1). The child survived initial palliation, and at 16 months of age underwent an atriopulmonary Fontan. The anomaly known as hypoplastic left heart syndrome (HLHS) is not rare. Norwood's initial report referenced the New England Regional Infant Cardiac Program that diagnosed 223 infants in New England alone over a 10 -year period-none of which survived to 1 year $(1,2)$. Norwood's comments at the conclusion of 
this article were prescient indeed, echoing the evolution of contemporary perspectives on HLHS care, "obviously, determination of the efficacy of such management must await long-term follow-up of a substantial group of patients." (1). While in-hospital mortality and morbidity for babies with HLHS have continued to improve, there remains a long road ahead to achieve ideal outcomes. Important variation in even these abbreviated in-hospital metrics persists among institutions and currently utilized prediction models explain only a small amount of this variation. Moreover, mid-term and long-term survival and neurodevelopmental health for patients with HLHS are infrequently reported and remain suboptimal despite improved in-hospital outcomes (3-8).

This focused review will describe the evolution of national outcomes for HLHS over time and the potential factors motivating improved time-related mortality. Emerging modifiable risk-factors that hold promise in terms of moving the needle for long-term success, including social determinants of health and the elucidation of genetic profiles, will be discussed. Specifically, this review will integrate contemporary data based on the first murine HLHS models that suggest a genetically elicited modular phenotype with environmental factors known to impact the initial durability of surgical therapies. A comprehensive approach to the management of HLHS, which leverages both proactive transplantation and hybrid palliation, in addition to traditional Norwood palliation, will be emphasized to extend and match management to the complete spectrum of patient risk-profiles. Finally, we will explore the critical role that national collaboratives and quality reporting initiatives have played in improving outcomes and shifting the focus to more meaningful longterm survival and neurodevelopment.

\section{Evolution of National Outcomes for HLHS}

How have results changed over time since the initial description of the Norwood procedure in 1980? Have changes in national practice patterns mirrored changes in survival? These are difficult questions to answer given the impact of institutional biases coupled with different reported outcome metrics. The Society of Thoracic Surgeons' Congenital Heart Surgery Database (STSCHSD) 2018 annual report documents Norwood operative mortality of $15.8 \%$, which is consistent with the $16 \%$ Norwood hospital mortality rate reported in the initial report of the SVR trial published in 2010 (9-11). These short-term survival statistics, though not ideal, represent a substantial improvement in the hospital survival reported in most early series that ranged from $52 \%$ to $68 \%$ (12). While the initial description of the Sano modification (13) held promise to decrease early attrition and adverse events ascribed to the frailty of shunted physiology, it remains unclear how widespread adoption of this technique has been and more importantly, how it is utilized at institutions with varied levels of expertise. Current research studies to describe these kinetics are needed, but longer term data from the latest publication from the SVR cohort (SVR II) that does not equivocally demonstrate a durable survival benefit, may again alter these dynamic endpoints.

\section{Beyond hospital mortality: shifting the focus to longer-term outcomes}

Returning to Norwood's prophetic statements about the importance of applying the long-term lens to contextualize early surgical success, most congenital heart disease (CHD) caregivers have recently emphasized time-related survival and neurodevelopmental quality of life for HLHS patients. Compilation of literature published between 2000-2005 consistently demonstrated 40-50\% 10-year survival, with modest advances around 2010 to $60-70 \%$ 10-year survival $(12,14)$. Importantly, the shape of the survival curves over time are highly conserved, with $90 \%$ of deaths clustered in the first year of life. Maturation of the SVR trial cohort has contributed significantly to our understanding of the fate of contemporary Norwood palliation $(5,7,10)$. SVR II showed, as expected, lower overall transplant-free survival at 6 years, which was insensitive to shunt type [ $64 \%$ for the right ventricle to pulmonary artery shunt (RVPAS) group compared to $59 \%$ for the modified Blalock-Taussig shunt (MBTS) group, $\mathrm{P}=0.13]$ (7). Importantly, the magnitude of the treatment effect on the primary endpoint (transplantfree survival) varied across the three intervals studied in this trial. The RVPAS group had a lower risk of death or transplant prior to stage II (HR 0.66; $\mathrm{P}=0.003$ ), with a trend toward increased risk of death during the second interval (HR 1.36; $\mathrm{P}=0.17)(3,6)$. In the third interval, shunt type was not significantly associated with transplant-free survival, although the hazard ratio was lower for the RVPAS group (HR 0.76; $\mathrm{P}=0.52$ ). The incidence of catheter interventions was higher among the RVPAS group $(4,7)$, predominantly because these patients underwent initial interventions at an earlier age following Norwood operation. Finally, serious adverse clinical events were prevalent among all survivors, with $20 \%$ having a neurologic event $(13 \%$ seizure and $7 \%$ 

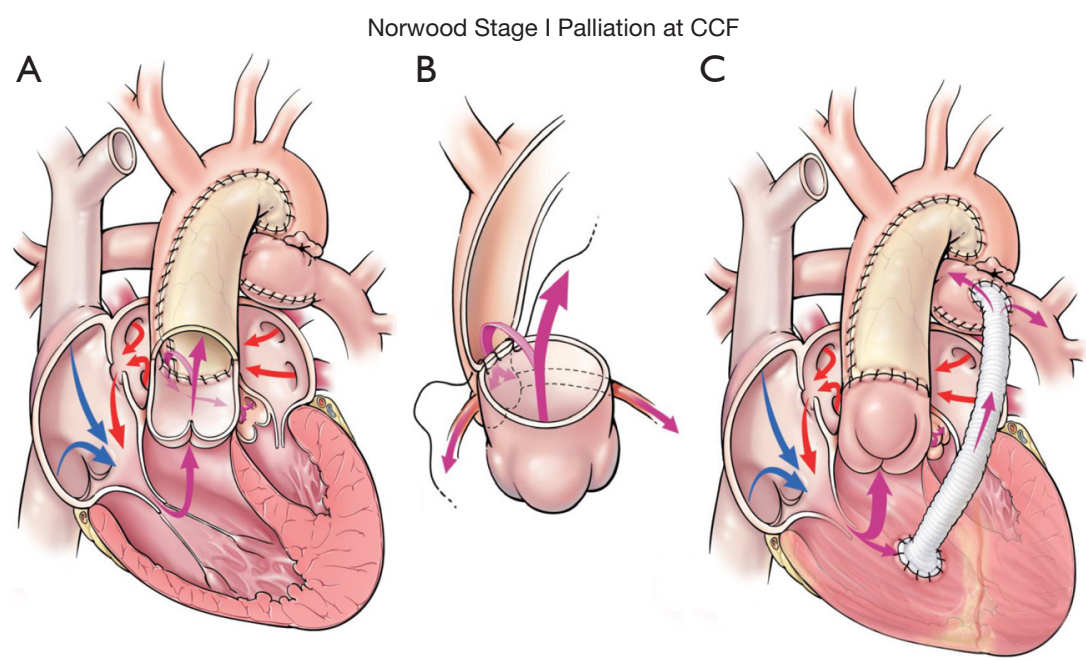

Figure 1 Norwood reconstruction at the Cleveland Clinic. (A) Arch reconstruction with patch; (B) aorta-PA anastomosis (Damus); (C) Sano modification: RV-PA conduit.

stroke), and nearly $16 \%$ having a thrombotic event. Timerelated survival in the latest multi-institutional report from the Congenital Heart Surgeons' Society (CHSS) cohort of infants with critical left heart obstruction undergoing Norwood procedure was equally poor, with reported 6-year overall survival of $70 \%$ for RVPAS and $55 \%$ for MBTS (8). Unlike the SVR data, however, RVPAS outperformed the MBTS during the first 1.5 years post-Norwood, with divergence early and nearly parallel trajectories thereafterpotentially suggesting that the late survival advantage for patients with RVPAS was attributable to mitigation of inter stage death in this group. Tweddell and colleagues (15) proposed one explanation for differences in the benefit of specific shunt type among contemporaneous prospective studies, suggesting that interactions between anatomic factors and shunt-type favored RVAPS for aortic valve atresia whereas the MBTS was favored with aortic valve stenosis.

Despite the potentially conflicting data on the superiority of one shunt type over another, it is our perception that the MBTS is more brittle and vulnerable to acute decompensation and that RVPAS allows for a transition to heart transplantation ( $\mathrm{Tx}$ ) for at risk patients. Patients with MBTS may experience unexpected, acute and irreversible decompensation that, coupled with higher interstage mortality, may reduce the likelihood of successful Tx. The acute benefits of RVPAS compared to MBTS have been elucidated by others $(11,13,16)$. Beyond the initial convalescent phase, however, it is likely that the more stable circulation afforded by lack of diastolic run-off, may result in higher physiologic tolerance (and therefore the probability of rescue) in the presence of hemodynamically significant lesions. Data from the SVR trial would support this contention. Hill and colleagues found that BT shunt type reduced early survival of infants with arch obstruction (17), and a greater proportion of patients in the RVPAS group were 'rescued' by Tx at 1-year (7 patients undergoing transplant in the RVPAS vs. 1 patient in the MBTS group). Ironically, the higher prevalence of Tx among RVPAS recipients can (and has) been interpreted by some as a relative condemnation of this configuration-a position that is facilitated by the adoption of transplant-free survival as the endpointtherefore equating transplantation with death.

Reducing re-intervention among HLHS patients for arch obstruction, which currently occurs in between 17-25\% of patients, is another critical area of contemporary focus $(17,18)$. Modifications of arch reconstruction technique to include complete coarctectomy have been suggested to reduce re-coarctation (17-19). Our current method for Norwood arch reconstruction is depicted in Figure 1. Interdigitation of the distal neoartic anastomosis has also been shown to reduce arch re-obstruction requiring reintervention (18). This technique includes the following maneuvers: (I) removal of all ductal tissue, including the periductal area; (II) creation of two longitudinal incisions in the anterior and posterior walls of the descending aorta; (III) construction of an extended end-to-end anastomosis 
between the distal aortic arch and the posterior descending aorta; (IV) a homograft patch is then used to reconstruct the remainder of the arch (18). Prospective investigation by our group of the role of retained ductal tissue from a mechanistic and computational fluid dynamics perspective may provide valuable insight to address this vexing complication. More importantly, uncovering reliable methods to identify retained ductal tissue intraoperatively may provide a real-time 'guide' to optimize arch reconstruction.

Neurodevelopmental outcomes have received considerable attention in recent years (20-22). Recent reports demonstrate that $59 \%$ of full-term neonates with CHD exhibit evidence of brain injury using conventional neuroimaging (20-22). Projections from a congenital cardiac surgery collaborative in the state of California (23) revealed that the cost of neurologic impairments in preterm infants nationally leads to over \$322 million/year in special educational services and that early detection and targeted intervention among neonates and infants with complex CHD in CA could result in annual savings of $\$ 4,040,318$ just for inpatient costs (23). Babies with HLHS are known to be at greatest risk for adverse neurologic outcomes for multifactorial reasons, including abnormal fetal development and perioperative injury (24-28). A large meta-analysis by Khalil and colleagues reported a prevalence of preoperative brain abnormalities, including ventriculomegaly, white matter injury, ischemic lesions, periventricular leukomalacia, and cerebral atrophy, in $49 \%$ of newborns with left-sided obstructive lesions (28). Many of these lesions are purported to be secondary to reduced antegrade flow in the aortic arch during fetal life, which may be proportional to the size of the ascending aorta (23-29). However, injuries sustained during the perioperative period, in which the neonate is subjected to significant hemodynamic impairment, altered perfusion, and extremes of temperature are also instrumental — and modifiable. While it would be ideal to intervene in the prenatal period to reduce the prevalence of preoperative brain injury among neonates with HLHS, ensuring that cerebral perfusion is optimized could mitigate neurologic risk, and dramatically improve neurodevelopmental outcome. The use of antegrade cerebral perfusion, in which continuous blood flow is maintained to the brain during arch reconstruction via an arterial cannula placed in the innominate artery, may represent one method to enhance neurologic protection. The STS-CHSD study of 4,523 neonatal arch repairs from Meyer and colleagues (30) showed that RCP was the most prevalent perfusion strategy, utilized in $43 \%$ of cases. Unfortunately, variability in practice was frequent and clear long-term data assessing the relative merits of RCP are still needed. Moreover, despite such advances in perfusion strategies and operative techniques, a recent prospective trial demonstrated that significant neurologic impairment was essentially unchanged from a prior era (27).

\section{Emerging risk-factors: nature vs. nurture-the genetic signature and social determinants of health}

Current variation in outcomes for HLHS remains largely unexplained by traditional risk-factors, including demographics (age, weight), anatomy, and treatmentspecific factors $(4,12,14,23,29)$. Most contemporary risk-models have $\mathrm{R} 2$ values less than 0.2 despite fervent searches for relationships, interactions, and inclusion of surgeon and institutional factors. Buoyed by increased appreciation of the genetic underpinnings of many complex congenital diseases, several investigators thought to apply old ideas, the importance of 'nature and nurture', to the puzzle of HLHS $(23,31)$. The wide morphologic spectrum that characterizes HLHS and its variants supports the notion that 'critical left ventricular outflow obstruction' would be mediated by a complex relationship between epigenetic and environmental influences. Fortunately, the development of a mouse model for HLHS coupled with application of systems biology, facilitated the discovery that HLHS is genetically heterogeneous and may arise in a modular fashion mediated by multiple genetic mutations that are conditioned by environmental factors $(32,33)$ In a recent report left ventricular hypoplasia was linked by Liu and colleagues (33) to intrinsic defects in cardiomyocyte proliferation and differentiation, suggesting that the HLHS phenotype is more than a flow-mediated aortic valve defect and likely arises in a combinatorial manner. These authors identified two candidate mutations in Sap130 and Pcdha9 as genetic causes of HLHS in which Sap130 drives LV hypoplasia by perturbing cardiomyocyte proliferation and differentiation, whereas protocadberin mutation drives the aortic valve defects. Other candidate mutations in core transcriptional factors regulating heart development that are highly conserved across species include NOTCH1, NKX2.5, and RbFOX2, and ETS 1 (34-36). Pioneering work by led by the Pediatric Cardiac Genomics Consortium and the Bench-to-Bassinet initiatives will undoubtedly lead to a more mechanistic understanding of pathobiologic drivers of left heart obstruction and the development of precision-based 
treatment algorithms for HLHS.

Given the genetic component, what are the environmental factors that condition the phenotypic response in HLHS?

Healthcare disparities that translate to reduced access to high-specialty care are well-known to adversely impact survival in children with chronic diseases $(23,36)$. The relatively higher acuity and longitudinal surveillance that defines the lives of HLHS patients undoubtedly increase the impact of socioeconomic and racial factors among these patients. In a post-hoc analysis of the SVR public use dataset, Bucholz and colleagues found that lower SES tertile was associated with 1-year mortality rates and that the hazard for death decreased linearly with increasing neighborhood SES (36). The experience with interstage monitoring clearly demonstrated a survival benefit when physiologically fragile patients without reliable home environments were managed in-hospital (31). Karamlou and colleagues investigated SES, racial factors, and distances traveled to quaternary centers of excellence in the Pediatric Health Information Systems Database and state administrative data (23) with very similar conclusions. Importantly, lower SES was associated with increased mortality, and also impacted the ability of patients to travel to centers for care. New initiatives to facilitate travel for government-sponsored (Medicaid) recipients with complex congenital diseases may address current healthcare inequities and increase access, but are unlikely to provide tenable solutions for patients who require continued surveillance over time. Scalable and pragmatic national frameworks that can provide continuity for socioeconomically or racially diverse patients will require more comprehensive models of care that contextualize social determinants in a multidimensional way rather than as individual determinants. That is to say, the socioeconomic profile of a patient or a 'family-unit' with HLHS is a complex ecosystem that is conditioned by both income level, race, maternal education, surrounding neighborhoods, and geographic region. It is probable that interactions among these factors will either magnify the risk, or neutralize the risk of an adverse outcome. Seeking inflection points for transitions to lower states of 'risk' is a promising avenue of research that can lead to targeted resource allocation and local, regional, or national health policy mandates.

\section{Reducing practice variation among and within centers}

Quality improvement (QI) science has shown consistent benefits when standardization of practice is employed in healthcare environments $(37,38)$. Reductions in mortality and morbidity as well as cost coupled with increased team efficacy, patient and provider safety have all been realized with the application of QI-based 'best-practices'. The landmark quality collaborative studies that unequivocally demonstrated proof of concept were reported by the Northern New England Cardiovascular Study Group. This unique partnership among participating institutions developed a structural framework for regional quality collaboratives in adult cardiac surgery, and elucidated the need to assess the degree of variation in hospital practice and outcomes, develop empirically based best practices, and implement guidelines across hospitals (37-39). The quest for value-based care metrics in congenital heart surgery, defined as outcomes relative to the patient over the costs for the cycle of care, are based on the results of these initial collaborative efforts and stem from the persistent and widespread variation in CHS care. Pertinent to the population of HLHS, data from the STS-CHSD $(4,40)$ suggests that suboptimal results following the Norwood procedure relates, in part, to the tremendous variability in practices across hospitals and surgeons. Pasquali and colleagues $(40,41)$ summarized this variability in Norwood hospitalization outcomes and practices, and also intimated that the lack of standardization may be a major factor impeding progress. She reported that, across the 15 SVR trial sites, in-hospital mortality ranged from $7-39 \%$, delayed sternal closure from $35-100 \%$, extubation within 72 hours of Norwood from $5-63 \%$ and percentage of patients discharged home on all oral feeds from $3 \%$ to $81 \%$. Furthermore, Oster and colleagues (42) demonstrated that this variability related to the postoperative Norwood management as well, specifically relating to the use of discharge digoxin among SVR trial patients; some centers discharged $100 \%$ of arrhythmia free patients on digoxin while others discharged $0 \%$ of these same patients. Importantly patients discharged on digoxin had a hazard ratio for mortality 3.5 -fold lower than those not discharged on digoxin. Leveraging another national quality collaborative effort, this time from the National Pediatric Cardiology Quality Improvement Collaborative (NPC-QIC), demonstrated persistent variability in the management of post-Norwood infants through Stage II $(4,40,42)$.

Inherent in the thought paradigm of iterative process improvement is the development of best practices. Unfortunately, because of small numbers and numerous 
surgical approaches, congenital heart surgery generally, and the Norwood procedure specifically, lacks standardized definitions for prevalent complications (such as arch obstruction), which confound the ability to study these outcomes among centers and establish meaningful performance benchmarks. Devlin and colleagues (18) utilized the CHSS left ventricular outflow tract obstruction cohort to investigate thresholds and approaches to arch obstruction following Norwood palliation. These authors found important institutional variability among 27 participating centers despite the high prevalence of arch interventions among patients $(25 \%)$. Pre-intervention arch gradient ranged from $0-64 \mathrm{mmHg}$, and even within the same institution, there was no correlation between arch intervention frequency and median pre-intervention gradient. This echoed findings from the study by Hill and colleagues (17) that evaluated rates of arch re-intervention (surgical or trans-catheter) for re-coarctation at or before stage II surgery, in which center level re-intervention rates ranged from $0 \%$ to $50 \%$ across the 15 SVR centers with very little consistency in terms of interventional thresholds. Importantly, institutional practice, or bias toward an interventional strategy, was associated with a higher prevalence of arch intervention regardless of arch gradient or other patient factors $(4,17,18)$.

Efforts to increase standardization have been championed by several national groups, most that have emphasized the importance of integrating multiple factors, including systemic ventricular function, overall clinical status, and the presence of other hemodynamically significant lesions, to inform the decision to proceed with an intervention. One effort that was spearheaded by the Pediatric Cardiac Critical Care Consortium $\left(\mathrm{PC}^{4}\right)$-PHN collaborative demonstrated that structured implementation of protocols based upon extrapolated study of high-performers, is a successful strategy to reduce variation and improve targeted outcomes among participants (43-45). Unfortunately, $75 \%$ of centers reverted to pre-trial performance, suggesting that ongoing efforts to revisit and redirect quality improvement initiatives are warranted to prevent attrition.

\section{Deployment of alternative therapies: proactive (primary?) transplantation, bybrid palliation}

Individualized care for HLHS, tailoring strategies to patient's genetic signature, characteristics, anatomy, and physiology may be one pathway to improved outcomes. Careful consideration of Initial patient-factors (including genetic or non-cardiac conditions) coupled with institutional or surgeon expertise (i.e., matching) should facilitate informed selection. The hybrid procedure is an example of a surgical innovation that was designed to reduce the risk of the Norwood procedure. First described by Akinteurk and colleagues in 2002 (46), the hybrid procedure involves bilateral pulmonary artery bands in conjunction with a ductal stent. More recently, centers have utilized prostaglandin to maintain ductal patency, either as a prolonged strategy or as a short-term initial sequence in the rapid-2-staged Norwood approach. Galantowicz et al. (47), in a review titled 'In Favor of the Hybrid Stage 1 as Initial palliation for HLHS', discussed a rationale for this possibility, “...hybrid techniques are especially useful in the following two scenarios: (I) when surgery or catheter-based interventions alone are not achieving a satisfactory result for a given problem, and (II) when the combination of the two fields results in less invasiveness and less trauma to the patient". Karamlou and colleagues (47) documented the early STS-CHSD experience with the Hybrid procedure in their analysis of 100 centers from 2010-2012. There was significant variation in adoption of this approach 'lag-time' kinetics that are seen with introduction of most innovative treatments or technology (47). During the 2 years of the study, 50 institutions never utilized the Hybrid procedure, and only 4 institutions utilized the Hybrid for $>90 \%$ of neonates. Patterns of Hybrid utilization included greater use in those institutions with lower HLHS case volume and higher Norwood mortality rates, suggesting that centers with higher Norwood mortality may preferentially utilize the hybrid as an alternative that can improve HLHS outcomes $(47,48)$.

Malik et al. (49) studied whether the Hybrid procedure is superior to the Norwood procedure for an unselected group of infants with HLHS. The Pediatric Health Information System database identified 3,654 neonates from 39 centers, of which 3,412 (93\%) underwent the Norwood and 242 (7\%) underwent the Hybrid. The vast majority of Hybrid procedures were performed at 5 hospitals, and 8 hospitals did not perform any hybrid procedures. The authors found that the use of the Hybrid procedure reduced the risk of mortality, renal failure, and decreased the index hospital length of stay by 20 days compared to the Norwood procedure $(49,50)$. Although the participating centers varied significantly in their utilization of the hybrid procedure, as found in the earlier study by Karamlou et al. (48), the validity of the results in the study by Malik et al. is reduced because important surgeon and center-level factors as well as the potential interaction of these factors were not 
considered in the analysis. Ultimately, whether the hybrid approach should be more widely applied is doubtful as centers with arguably excellent Norwood survival and proven durability should continue to refine, develop, and extrapolate those techniques. Historically, the enthusiasm for new surgical technology has often outpaced solid evidence of its benefits. Although the hybrid may offer advantages in select circumstances, we have not made much progress in elucidating these circumstances. Moreover, rapid adoption before such clarity and efficacy have been established is fiscally irresponsible and could adversely affect the fate of our most fragile patients (50).

The question about transplantation timing and the wisdom of primary transplantation for specific subtypes of HLHS (i.e., those with coronary sinusoids, obstructed atrial septum, compromised systemic ventricular function etc.) continues to be debated. Recent literature has suggested a modification of the endpoints considered in the SVR trial to include overall survival in lieu of transplant-free survival (4). Overall survival rather than transplant-free survival may be a more complete metric of longer-term center performance because transplant may eventually be required in many HLHS patients, with timing for transplant deployment a critical factor. One philosophy is to aggressively manage correctable hemodynamic lesions and if ineffective or unlikely to be successful, to transition patients to transplant proactively. Recent data from the SVR trial (7) demonstrated significant ongoing attrition beyond Stage 2 palliation. Similar trends were observed in the paper from the Congenital Heart Surgeons' Society $(6,8)$, which investigated the optimal timing of Stage 2 palliation among a cohort undergoing Norwood operation for critical left heart obstruction. Highrisk patients had markedly increased mortality following Stage 2 palliation (28\% compared to $2 \%$ for the low-risk cohort). Moreover, triaging these patients to earlier stage 2 resulted in reduced survival. Data from this large prospective multi-institutional series therefore suggested that early referral to transplant would be the preferred strategy to decrease mortality (6). Other studies have demonstrated that outcomes for transplant following Norwood palliation are generally encouraging, despite waitlist attrition and that proactive transition to this pathway may be beneficial in improving long-term outcomes and salvaging patients who are unexpected to have long-term Fontan durability.

The increased focus on neurodevelopmental outcomes and health-related quality of life among infants with HLHS also influenced our bias toward more proactive use of cardiac transplantation in the recent era. Currently, neurodevelopmental delay has been recorded in from $49-58 \%$ of those undergoing Norwood palliation $(7,51)$. The initial reports from the SVR trial demonstrated mean psychomotor developmental index scores of $74 \pm 19$, and mean mental developmental index scores of $89 \pm 18$, which were significantly lower compared to normative means $(4,7,51)$. These neurodevelopmental impairments persisted at repeat examination of the SVR cohort at 3 years post-Norwood palliation (18). Although long-term data concerning neurologic status among infants with primary transplantation is limited, Chinnock et al. (52) reported neurologic abnormalities in only $10 \%$ of the 142 infants undergoing primary transplantation in the early Loma Linda experience, and normal mental developmental and psychomotor indices among the majority of survivors. Later surveillance of infants transplanted at Loma Linda before the age of 6 months (median follow-up 13 years posttransplant) also demonstrated slightly delayed psychomotor development with normal cognitive development among survivors (53). Despite the benefits that the availability of cardiac transplantation provides, and the data from Jacobs et al. (54) suggesting that primary transplantation may lead to a survival benefit compared to post-Norwood transplantation, there are important limitations that must be carefully weighed. Limited supply of donor hearts is a critical issue. Also, it is unlikely that every center will have the capabilities to provide heart transplantation. These caveats illustrate the need to elucidate better riskstratification algorithms for HLHS patients (i.e., understand which patients may benefit from primary transplant) and triage them to the most appropriate initial strategy.

\section{Conclusions}

In summary, optimizing outcomes for patients with HLHS will be an ongoing and formidable challenge for our specialty. Collectively, we have come far in realizing shortterm benefits, but long-term quality of life and the promise of a 'normal' life for these babies remain elusive. Harnessing collaborative learning models and partnerships with basic scientists, innovators, and our patients and families to develop new paradigms and rediscover old ones should form the basis for progress. Would it be too clichéd to say "Let's do this?".

\section{Acknowledgments}

Funding: None. 


\section{Footnote}

Provenance and Peer Review: This article was commissioned by the Guest Editor (Raghav A. Murthy) for the series "Management of Congenital Heart Disease" published in Fournal of Thoracic Disease. The article was sent for external peer review organized by the Guest Editor and the editorial office.

Conflicts of Interest: The series "Management of Congenital Heart Disease" was commissioned by the editorial office without any funding or sponsorship. The authors have no other conflicts of interest to declare.

Ethical Statement: The authors are accountable for all aspects of the work in ensuring that questions related to the accuracy or integrity of any part of the work are appropriately investigated and resolved.

Open Access Statement: This is an Open Access article distributed in accordance with the Creative Commons Attribution-NonCommercial-NoDerivs 4.0 International License (CC BY-NC-ND 4.0), which permits the noncommercial replication and distribution of the article with the strict proviso that no changes or edits are made and the original work is properly cited (including links to both the formal publication through the relevant DOI and the license). See: https://creativecommons.org/licenses/by-nc-nd/4.0/.

\section{References}

1. Norwood WI, Lang P, Hansen DD. Physiologic repair of aortic atresia-hypoplastic left heart syndrome. N Engl J Med 1983;308:23-6.

2. Fyler DC. Report of the New England regional Infant Cardiac program. Pediatrics 1980;65:375-461.

3. Rogers L, Pagel C, Sullivan ID, et al. Interventional treatments and risk-factors in patients born with hypoplastic left heart syndrome in England and Wales from 2000 to 2015. Heart 2018;104:1500-7.

4. Karamlou T, Meza JM, Najm HK, et al. Single ventricle reconstruction 2.0: A closer look at our understanding of care for infants undergoing the Norwood procedure. J Thorac Cardiovasc Surg 2019;158:837-42.e5.

5. Meza JM, Hickey EJ, Blackstone EH, et al. The optimal timing of stage 2 palliation for hypoplastic left heart syndrome: an analysis of the pediatric heart network single ventricle reconstruction trial public data set. Circulation
2017;136:1737-48.

6. Meza JM, Hickey E, McCrindle B, et al. The optimal timing of stge-2-palliation after the Norwood operation. Ann Thorac Surg 2018;105:193-9.

7. Newburger JW, Sleeper LA, Frommelt PC, et al. Transplantation-free survival and interventions at 6 years in the Single Ventricle Reconstruction Trial. Circulation 2018; 137:2246-53.

8. Wilder TJ, McCrindle BW, Phillips AB, et al. Survival and right-ventricular performance for matched children after stage 1 Norwood: Modified Blalock-Taussig shunt versus right-ventricle-to-pulmonary -artery conduit. J Thorac Cardiovasc Surg 2015;150:1440-50.

9. Jacobs JP, Shahian DM, D'Agostino RS, et al. The Society of Thoracic Surgeons National Database 2018 Annual Report. Ann Thorac Surg 2018;106:1603-11.

10. Ohye RG, Schonbeck JV, Eghtesady P, et al. Cause, timing, and location of death in the Single Ventricle Reconstruction trial. J Thorac Cardiovasc Surg 2012;144:907-14.

11. Ohye RG, Sleeper LA, Mahony L, et al. Comparison of shunt types in the Norwood procedure for single ventricle lesions. N Engl J Med 2010;362:1980-92.

12. DiBardino DJ. Long-term progression and survival following Norwood single ventricle reconstruction. Curr Opin Cardiol 2015;30:95-9.

13. Sano S, Ishino K, Kado H, et al. Outcome of rightventricle-to-pulmonary artery shunt in first stage palliation of hypoplastic left heart syndrome: a multi-institutional study. Ann Thorac Surg 2004;78:1951-7.

14. Greenleaf CE, Urencio JM, Salazar J, et al. Hypoplastic left heart syndrome: current perspecives. Transl Pediatr 2016;5:142-47.

15. Tweddell JS, Sleeper LA, Ohye RG, et al. Intermediateterm mortality and cardiac transplantation in infants with single-ventricle lesions: risk-factors and their interaction with shunt type. J Thorac Cardiovasc Surg 2012;144:152-9.

16. Ghanayem NS, Jaquiss RD, Cava JR, et al Right ventricle-to-pulmonary artery conduit versus BT shunt: a hemodynamic comparison. Ann Thorac Surg 2006;82:1603-9.

17. Hill KD, Rhodes JF, Aiyagari R, et al. Intervention for recoarctation in the single ventricle reconstruction trial: incidence, risk, and outcomes. Circulation 2013;128:954-61.

18. Devlin PJ, McCrindle BW, Kirklin JK, et al. Intervention for arch obstruction after the Norwood procedure: prevalence, associated factors, and practice variability. J 
Thorac Cardiovasc Surg 2019;157:684-95.e8.

19. Bautista-Hernandez V, Marx GR, Gauvreau K, et al. Coarctectomy reduces neoaortic arch obstruction in hypoplastic left heart syndrome. J Thorac Cardiovasc Surg 2007;133:1540-6.

20. Brosig C, MUssatto K, Hoffman RG, et al. Neurodevelopmental outcomes for children with hypoplastic left heart syndrome at the age of 5 years. Pediatr Cardiol 2013;34:1597-604.

21. Mahle WT, Lu M, Ohye RG, et al. A predictive model for neurodevelopmental outcome after the Norwood procedure. Pediatr Cardiol 2013;34:327-33.

22. Newburger JW, Sleeper LA, Bellinger DC, et al. Early developmental outcome in children with hypoplastic left heart syndrome and related anomalies: the single ventricle reconstruction trial. Circulation 2012;125:2081-91.

23. Karamlou T, Peyvandi S, Federman M, et al. Resolving the Fontan paradox: addressing socioeconomic and racial disparities in patients with single ventricle. J Thorac Cardiovasc Surg 2018;155:1727-31.

24. Yamamoto Y, Khoo NS, Brooks PA, et al. Severe left heart obstruction with retrograde arch flow influences fetal cerebral and placental blood flow. Ultrasound Obstet Gynecol 2013;42:294-9.

25. Hahn E, Szwast A, Cnota II J, et al. Association between fetal growth, cerebral blood flow and neurodevelopmental outcome in univentricular foetuses. Ultrasound Obstet Gynecol 2016;47:460-5.

26. Gunn JK, Beca J, Hunt RW, et al. Perioperative risk factors for impaired neurodevelopment after cardiac surgery in infancy. Arch Dis Child 2016;101:1010-6.

27. Sarajuuri A, Jokinen E, Mildh L, et al. Neurodevelopmental burden at age 5 years in patients with univentricular heart. Pediatrics 2012;130:e1636-46.

28. Khalil A, Suff N, Thilaganathan B, et al. Brain abnormalities and neurodevelopmental delay in congenital heart disease; systematic review and meta-analysis. Ultrasound Obstet Gynecol 2014;43:14-24.

29. Gaynor JW, Wernovsky G, Jarvik GP, et al. Patient characteristics are important determinants of neurodevelopmental outcome at one year od age after neonatal and infant cardiac surgery. J Thorac Cardiovasc Surg 2007;133:1344-53.

30. Meyer DB, Jacobs JP, Hill K, et al. Variation in perfusion strategies for neonatal and infant aortic arch repair: contemporary practice in the STS Congenital Heart Surgery Database. World J Pediatr Congenit Heart Surg 2016;7:638-44.
31. Bucholz EM, Sleeper LA, Newburger JW. Neighborhood socioeconomic status and outcomes following Norwood procedure: Ananalysis of the pediatric Heart Network Single Ventricle Reconstruction Trial Public Data set. J Am Heart Assoc 2018;7:e007065.

32. Saraf A, Book WM, Nelson TJ, et al. Hypoplastic left heart syndrome: From bedside to bench and back. J Mol Cell Cardiol 2019;135:109-18.

33. Liu X, Yagi H, Saeed S, et al. The complex genetics of hypoplastic left heart syndrome. Nat Genet 2017;49:1152-59.

34. Esposito G, Butler TI, Blue GM, et al. Somatic mutations in NKX205, GATA4, and HAND1 are not a common cause of tetralogy of Fallot or hypoplastic left heart. Am J Med Genet 2011;155A:2416-21.

35. Liu X, Francis R, Kim AJ, et al. Interrogating congenital heart defects with noninvasive fetal echocardiography in a mouse forward genetic screen. Circ Cardiovasc Imaging 2014;7:31-42.

36. Yagi H, Liu X, Gabriel GC, et al. The genetic landscape of hypoplastic left heart syndrome. Pediatr Cardiol 2018;39:1069-81.

37. Ghanayem NS, Allen KR, Tabbutt S, et al. Interstage mortality after the Norwood procedure. Results of the multicenter Single Ventricle Reconstruction trial. J Thorac Cardiovasc Surg 2012;144:896-906.

38. Likosky DS. Lessons learned from the Northern New England Cardiovascular Disease Study Group. Prog Pediatr Cadriol 2012;33:53-6.

39. Anderson JB, Beekman RH, Kugler JD, et al. Improvement in interstage survival in a National Pediatirc Cardiology Learning Network. Circ Cardiovasc Qual Outcomes 2015;8:428-36.

40. Pasquali SK. Re-examining interstage home monitoring following the Norwood operation. Circulation 2015;132:475-8.

41. Pasquali SK, Ohye RG, Lu M, et al. Variation in perioperative care across centers for infants undergoing the Norwood procedure. J Thorac Cardiovasc Surg 2012;144:915-21.

42. Oster ME, Kelleman M, McCracken C, et al. Association of digoxin with interstage mortality: results from the Pediatric Heart Network Single Ventricle Reconstruction Trial Public Use Dataset. J Am Heart Assoc 2016;5:1.

43. Baker-Smith CM, Neish SR, Klitzner TS, et al. Variation in postoperative care following stage I palliation for singleventricle patients: a report from the Joint Council on 
Congenital Heart Disease National Quality Improvement Collaborative. Congenit Heart Dis 2011;6:116-27.

44. Gaies M, Pasquali SK, Nicolson SC, et al. Sustainability of infant cardiac surgery early extubation practices after implementation ANS study. Ann Thorac Surg 2019;107:1427-33.

45. Karamlou T, Rao R, Najm HK. Collaborative learning models in pediatric cardiac care: the continuum of learning and positive change through shared inquiry. Ann Thorac Surg 2019;107:1293-6.

46. Akintuerk H, Michel-Behnke I, Valeske K, et al. Stenting of the arterial duct and banding of the pulmonary arterties: basis for combined Norwood stage I and II repair in hypoplastic left heart. Circulation 2002;105:1099-103.

47. Galantowicz M, Cheatham JP, Phillips A, et al. Hybrid approach for hypoplastic left heart syndrome: intermediate results after the learning curve. Ann Thorac Surg 2008;85:2063-70.

48. Karamlou T, Overman D, Hill KD, et al. Stage 1 hybrid palliation for hypoplastic left heart syndrome- assessment of contemporary use patterns: an analysis of the Society of
Thoracic Surgeons Congenital Heart Surgery Database. J Thorac Cardiovasc Surg 2015;149:195-201.

49. Malik S, Bird TM, Jaquiss RDB, et al. Comparison of in-hospital and longer-term outcomes of hybrid and Norwood stage 1 palliation of hypoplastic left heart syndrome. J Thorac Cardiovasc Surg 2015;150:474-80.e2.

50. Cua CL, McConnell PI, Meza JM, et al. Hybrid palliation: outcomes after the comprehensive stage 2 procedure. Ann Thorac Surg 2018;105:1455-60.

51. Karamlou T. Hybrid vs. Norood: "Fifty shades of grey." J Thorac Cardiovasc Surg 2015;150:456-7.

52. Chinnock RE, Bailey LL. Heart transplantation for congenital heart disease in the first year of life. Curr Cardiol Rev 2011;7:72-84.

53. Goldberg CS, Lu M, Sleeper LA, et al. Factors associated with neurodevelopment for children with single ventricle lesions. J Pediatr 2014;165:490-6.e8.

54. Jacobs JP, Quintessenza JA, Chai PJ, et al. Rescue cardiac transplkantation for failing staged palliation in patients with hypoplastic left heart syndrome. Cardiol Young 2006;16:556-62.
Cite this article as: Karamlou T, Najm HK. Evolution of care pathways for babies with hypoplastic left heart syndrome: integrating mechanistic and clinical process investigation, standardization, and collaborative study. J Thorac Dis 2020;12(3):1174-1183. doi: 10.21037/jtd.2019.10.75 